\title{
Morbidity and Mortality of Crystalloids Compared to Colloids in Critically III Surgical Patients
}

\section{A Subgroup Analysis of a Randomized Trial}

\author{
Nicholas Heming, M.D., Ph.D., Laure Lamothe, M.D., Samir Jaber, M.D., Ph.D., Jean Louis Trouillet, M.D., \\ Claude Martin, M.D., Ph.D., Sylvie Chevret, M.D., Ph.D., Djillali Annane, M.D., Ph.D.
}

\section{ABSTRACT}

Background: The multicenter randomized Colloids versus Crystalloids for the Resuscitation of the Critically Ill (CRISTAL) trial was designed to test whether colloids altered mortality compared to crystalloids in the resuscitation of intensive care unit patients with hypovolemic shock. This preplanned analysis tested the same hypothesis in the subgroup of surgical patients.

Methods: The CRISTAL trial prospectively defined patients as critically ill surgical patients whenever they underwent emergency or scheduled surgery immediately before or within $24 \mathrm{~h}$ of intensive care unit admission and had hypovolemic shock. The primary outcome measure was death by day 28 . Secondary outcome measures included death by day 90 , the need for renal replacement therapy, or the need for fresh frozen plasma transfusion.

Results: There were 741 critically ill surgical patients, 356 and 385 in the crystalloid and colloid arm, respectively. Median (interquartile range) age was $66(52$ to 76$) \mathrm{yr}$, and 484 (65.3\%) patients were male. Surgery was unscheduled in 543 (73.3\%) cases. Mortality by day 28 did not significantly differ for crystalloids 84 (23.6\%) versus colloids 100 (26\%; adjusted odds ratio, 0.86; $95 \%$ CI, 0.61 to $1.21 ; P=0.768)$. Death by day $90(111$ [31.2\%] vs. 122 [31.7\%]; adjusted odds ratio, 0.97; 95\% CI, 0.70 to $1.33 ; P=0.919)$ did not significantly differ between groups. Renal replacement therapy was required for $42(11.8 \%)$ patients in the crystalloids arm versus $49(12.7 \%)$ in the colloids arm $(P=0.871)$.

Conclusions: The authors found no survival benefit when comparing crystalloids to colloids in critically ill surgical patients.

$\mathbf{P}$

ERIOPERATIVE hemodynamic instability may lead to cardiovascular morbidity and requires prompt recognition and correction. Possible causes include blood loss, fluid deficit, or sepsis. Fluid therapy is therefore a key component of the perioperative management of surgical patients. Resuscitation fluids are divided into two categories: colloid and crystalloid solutions. The ideal fluid to be used in the surgical setting remains uncertain. ${ }^{1,2}$ Colloids are composed of heavy molecular weight molecules, which are retained in the plasma compartment. Hemodynamic goals are reached by administrating smaller volumes of colloids than crystalloids. ${ }^{3-5}$ Among colloids, starches are the most commonly administered fluid. The use of starches has been restricted by the European Medicines Agency in sepsis, burns, or critically ill patients ${ }^{6}$ because of the risk of acute kidney injury and of death. ${ }^{5,7}$ The U.S. Food and Drug Administration also issued a warning about the increased risk of renal failure or death,

- Whether crystalloid or colloids are preferable for treatment of hypovolemic shock in surgical patients remains unclear

- In a preplanned subgroup analysis of a previous trial, the authors compared 28-day mortality in 741 surgical patients with hypovolemic shock who were randomized to crystalloids or colloids

- Mortality at 30 and 90 days was similar in the two groups, and colloid administration did not increase the need for dialysis

- Colloid administration did not improve mortality but also did not cause renal injury

as well as a risk of bleeding after cardiopulmonary bypass associated with starches. ${ }^{6}$ However, because these data do not derive exclusively from surgical patients, extrapolation

Supplemental Digital Content is available for this article. Direct URL citations appear in the printed text and are available in both the HTML and PDF versions of this article. Links to the digital files are provided in the HTML text of this article on the Journal's Web site (www.anesthesiology.org). Part of the work presented in this article has been presented at the 46th French Intensive Care Society Congress in Paris, France, January 24 to $26,2018$. 
of these findings to the perioperative period is questionable. Indeed, crystalloids are not devoid of side effects such as hyperchloremic metabolic acidosis, reduced renal blood flow, or impaired renal cortical perfusion. ${ }^{8,9}$

The Colloids versus Crystalloids for the Resuscitation of the Critically Ill (CRISTAL) trial was designed to test the hypothesis that colloids altered 28-day mortality compared with crystalloids for fluid resuscitation in a general population of critically ill patients. ${ }^{4}$ This a priori defined secondary analysis tested the same hypothesis in the subgroup of surgical critically ill patients. Patients were identified as surgical whenever they underwent emergency or scheduled surgery immediately before or within $24 \mathrm{~h}$ of intensive care unit admission. Our primary outcome was 28 -day mortality. Secondary outcomes included the occurrence of organ dysfunction over a 28-day period, as well as the need for renal replacement therapy, secondary surgical intervention, blood product administration, intensive care unit and hospital length of stay, and 90-day mortality.

\section{Materials and Methods}

\section{Study Setting and Patients}

The CRISTAL trial (ClinicalTrials.gov NCT00318942) randomly assigned 2,857 acutely hypovolemic patients from 57 participating centers in Europe, North Africa, and North America to receive either crystalloids or colloids. ${ }^{4}$ The study protocol was approved by local institutional review boards. Deferred written informed consent was obtained from participants or legally authorized surrogates. Included participants had not previously received any fluid in the intensive care unit and required fluid resuscitation for acute hypovolemia. Acute hypovolemia was defined by the combination of (1) hypotension: systolic arterial pressure of less than $90 \mathrm{mmHg}$, mean arterial pressure of less than $60 \mathrm{mmHg}$, orthostatic hypotension (i.e., decrease in systolic arterial pressure of at least $20 \mathrm{mmHg}$, from the supine to the semirecumbent position), or a delta pulse pressure of $13 \%$ or higher; (2) evidence for low filling pressures and low cardiac index, assessed either invasively or noninvasively; and (3) signs of tissue hypoperfusion or hypoxia, including at least two of the following clinical symptoms: Glasgow Coma Scale score of less than 12, mottled skin, urinary output of less than $25 \mathrm{ml} / \mathrm{h}$, or capillary refilling time of $3 \mathrm{~s}$ or longer; and arterial lactate levels higher than $2 \mathrm{mM}$, blood urea nitrogen higher than $56 \mathrm{mg} / \mathrm{dl}$, or a fractional excretion of sodium of less than $1 \%{ }^{4}$

A computer-generated list with fixed-block permutation $(\mathrm{n}=4)$ was used to randomize patients on a 1 to 1 ratio. Randomization was stratified by center and by three admission diagnoses: sepsis, multiple trauma, and other causes of hypovolemic shock. Allocation concealment used sealed envelopes at the bedside to allow randomization of eligible patients without any delay. Investigators were blinded to block size.
Eligible patients were randomly allocated to fluid resuscitation with crystalloids or with colloids. In the crystalloids group, allowed treatments included isotonic or hypertonic saline and any buffered solutions. In the colloids group, hypooncotic (e.g., gelatins, and 4 or $5 \%$ of albumin) and hyperoncotic (e.g., dextrans, hydroxyethyl starches, and 20 or $25 \%$ of albumin) solutions were permitted. Within each treatment group, investigators could use whichever fluids were available at their institution. The amount of fluid and duration of treatment was left at the discretion of the investigators with the following restrictions: (1) the daily total dose of hydroxyethyl starch could not exceed $30 \mathrm{ml} /$ $\mathrm{kg}$ of body weight and (2) investigators were required to follow any local regulatory agency recommendations governing use. Patients had to be managed according to their randomization arm except for (1) maintenance fluids, which were isotonic crystalloids, regardless of treatment group, and (2) in instances in which physicians wished to administer albu$\mathrm{min}$ in response to demonstrated hypoalbuminemia (serum albumin concentration less than $20 \mathrm{~g} / \mathrm{dl}$ ).

The blinding of the clinicians to the fluid interventions was considered by the study advisors to be inappropriate or infeasible because study treatments had to be available immediately for resuscitation to ensure avoidance of nonstudy fluids in emergent situations. In addition, because the intervention would be continued until intensive care unit discharge and could thus be highly variable, there was no practical way to stock sites with adequate supplies of masked fluid solutions. However, the mortality endpoints were collected and assessed by study members blinded to treatment assignment. Similarly, the principal investigator, study sponsor, and the members of the data and safety monitoring board remained blinded to the study interventions until all patients were followed up and the final analysis was executed.

For this analysis, we included all surgical patients included in the original trial. Surgical patients were a priori defined as patients requiring elective or unscheduled surgery, either before or up to $24 \mathrm{~h}$ after intensive care unit admission.

\section{Data Collection}

At the time of randomization, age, sex, cause of intensive care unit admission, type of admission (medical, elective surgery, unscheduled surgery, trauma), McCabe class, ${ }^{10}$ disability scale score, ${ }^{11}$ cause of hypovolemia (divided into three separate strata: sepsis, trauma, and other), Simplified Acute Physiology Score II, ${ }^{12}$ Sequential Organ Failure Assessment score, ${ }^{13}$ Injury Severity Score, ${ }^{14}$ signs of hypovolemia, and amount of fluids administered before randomization were collected. The global Sequential Organ Failure Assessment score was recorded daily over a 7-day period and thereafter on days 14 and 28. Any occurrence of renal replacement therapy was recorded. We assessed the need for secondary surgical intervention and for blood product administration (including platelets, fresh frozen plasma, and packed erythrocytes) over a 7-day period. Outcomes included intensive 
care unit and hospital length of stay, as well as death by days 28 and 90.

\section{Statistical Analysis}

Quantitative variables were expressed as median (interquartile range) and categorical variables as number (percentage). When designing the CRISTAL trial, we anticipated that responses to colloids versus crystalloids may vary across different groups of patients, namely sepsis, trauma, and other categories of acute hypovolemia. Thus, randomization was stratified according to these three groups of patients. In addition, we anticipated potential qualitative interaction between treatment responses within each of these strata and the type of admission, namely surgical versus medical. Thus, we planned to report the estimation of treatment effects in the surgical and medical groups of patients separately. We undertook an intention-to-treat analysis for the primary outcome, death by day 28 , where patients, once selected in one treatment group according to randomization, were analyzed in the group assigned by the randomization, insuring the absence of any selection or attrition bias. No imputation was used. Nevertheless, in response to peer review, per-protocol analyses were added as a secondary analysis including all participants who adhered adequately to the assigned treatment. Categorical variables were compared with the Fisher exact test, and continuous variables were compared with the Wilcoxon rank sum test. To assess differences over time of the Sequential Organ Failure Assessment score across both arms, we built a linear mixed-effects model. This allowed us to model observational heterogeneity incurred by repeat measurements of the score in the same patient (with fixed effects of Sequential Organ Failure Assessment and time) and accounted for the fact that some individuals may have higher values than others (by using a random intercept).

All patients were followed until day 90 unless death occurred before day 90, so that analysis of mortality data across randomized groups used the chi-square test; estimate of odds ratio of death according to fluid used a logistic model, adjusted to the nature of surgery. To display the cumulative incidence of death, we used nonparametric estimator and then compared between randomized groups by the Gray test.

All analyses were preplanned, except for those factors selected for adjustment and additional analyses requested by the reviewers or editors. Statistical analyses were performed with SAS 9.3 (SAS Inc., USA) and R 2.13.0 (http:// www.R-project.org/; accessed August 10, 2017) software. Tests were two-sided. The results were adjusted for multiple comparisons. ${ }^{15} P$ levels less than 0.05 were considered statistically significant.

\section{Results}

\section{Baseline Characteristics}

Of 2,857 patients in the initial trial, there were 741 critically ill surgical patients (fig. 1). Of those 741 surgical patients, 484 (65.3\%) were male, 369 (49.8\%) suffered from sepsis, and the median age was 66 (52 to 76) yr. In total, 356 patients $(48 \%)$ were allocated to the crystalloids arm, and $385(52 \%)$ were allocated to the colloids arm. Surgery was elective for 198 (26.7\%) patients and unscheduled for 543

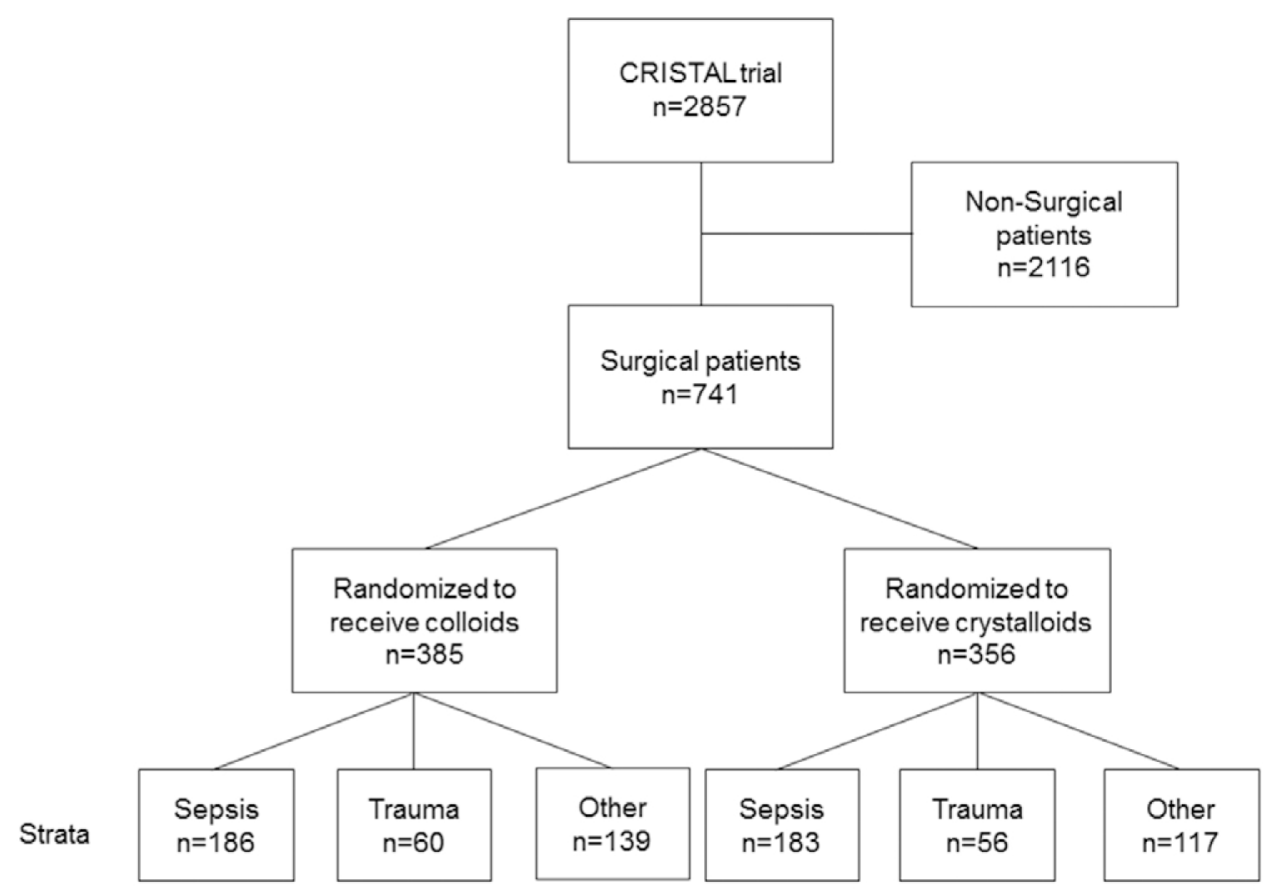

Fig. 1. Enrollment, randomization, and predetermined strata. 
(73.3\%) patients; 395 patients $(53.3 \%)$ underwent general or abdominal surgery, 198 patients $(26.7 \%)$ underwent orthopedic surgery, 127 patients (17.1\%) underwent cardiac surgery, and 21 patients $(2.9 \%)$ underwent neurosurgery. In both arms, some patients received crystalloids and/ or colloids in the operating theater before intensive care unit admission and before randomization (table 1). Nevertheless, severe acute hypovolemia was present upon randomization as highlighted by tachycardia, low systolic blood pressure and high diastolic blood pressure, low cardiac index, and high arterial lactate levels (Supplemental Digital Content, supplemental table 1, http://links.lww.com/ALN/B772).

\section{Outcomes}

Patients in the crystalloids arm received a median 7-day cumulative dose of fluids of 4,275 (2,000 to 7,500) $\mathrm{ml}$ versus $2,750(1,500$ to 4,500$) \mathrm{ml}$ for patients in the colloids arm (table 2). By day 1, the amount of fluids was markedly $(P<0.0008)$ higher in the crystalloids than in the colloidstreated patients (fig. 2). Over time, $14.8 \%(\mathrm{n}=57)$ of patients in the colloids arm received crystalloids, whereas 5.6\% $(\mathrm{n}=$ 20) of patients in the crystalloids arm received colloids.

There was no difference in the occurrence of death by day 28 between groups: $84(23.6 \%)$ in the crystalloids arm compared to $100(26 \%)$ patients in the colloids arm (adjusted

Table 1. Main Characteristics at Baseline According to Randomization Arm

\begin{tabular}{|c|c|c|}
\hline & $\begin{array}{l}\text { Colloids Arm } \\
(n=385)\end{array}$ & $\begin{array}{l}\text { Crystalloids Arm } \\
(n=356)\end{array}$ \\
\hline Age, median (IQR), yr & $65(52-76)$ & $67(52-76)$ \\
\hline Male sex, $n(\%)$ & $249 / 385(64.7)$ & $235 / 356(66.0)$ \\
\hline \multicolumn{3}{|l|}{ Reason for ICU admission, n (\%) } \\
\hline Scheduled surgery & $109 / 385(28.3)$ & $89 / 356(25.0)$ \\
\hline Emergency surgery & $276 / 385(71.7)$ & $267 / 356(75.0)$ \\
\hline \multicolumn{3}{|l|}{ Source of admission to ICU, $\mathrm{n}(\%)$} \\
\hline Community & $158 / 385(41.0)$ & $156 / 356(43.8)$ \\
\hline Hospital ward & $199 / 385(51.7)$ & $175 / 356(49.2)$ \\
\hline Other ICU & $15 / 385(3.9)$ & $18 / 356(5.0)$ \\
\hline Long-term care facility & $13 / 385(3.4)$ & $7 / 356(2.0)$ \\
\hline \multicolumn{3}{|l|}{ Type of surgery } \\
\hline General surgery & $200 / 385(51.9)$ & $195 / 356(54.8)$ \\
\hline Orthopedic surgery & $102 / 385(26.5)$ & $96 / 356(27.0)$ \\
\hline Cardiac surgery & $72 / 385(18.7)$ & $55 / 356(15.4)$ \\
\hline Neurosurgery & $11 / 385(2.9)$ & $10 / 356(2.8)$ \\
\hline \multicolumn{3}{|l|}{ McCabe class, n (\%) } \\
\hline No underlying disease or no fatal disease & 215/383 (56.1) & $210 / 356(59.0)$ \\
\hline Underlying ultimately fatal disease (> $5 \mathrm{yr}$ ) & 23/383 (6.0) & 13/356 (3.6) \\
\hline Underlying rapidly fatal disease $(<1 \mathrm{yr})$ & 145/383 (37.9) & $133 / 356(37.4)$ \\
\hline \multicolumn{3}{|l|}{ Knaus disability scale, $\mathrm{n}(\%)$} \\
\hline A & 94/383 (24.5) & $89 / 356(25.0)$ \\
\hline $\mathrm{B}$ & $139 / 383(36.3)$ & $133 / 356(37.4)$ \\
\hline $\mathrm{C}$ & $91 / 383(23.8)$ & $83 / 356(23.3)$ \\
\hline $\mathrm{D}$ & $59 / 383(15.4)$ & $51 / 356(14.3)$ \\
\hline Glasgow Coma Scale score, median (IQR) & $13(4-15)$ & $13(5-15)$ \\
\hline Simplified Acute Physiology Score II, median (IQR) & $45(30-62)$ & $47(33-66)$ \\
\hline $\begin{array}{l}\text { Sequential Organ Failure Assessment score, } \\
\text { median (IQR) }\end{array}$ & $7(4-11)$ & $7(5-11)$ \\
\hline Injury Severity Score, median (IQR) & $22(16-29)$ & $24(17-34)$ \\
\hline \multicolumn{3}{|l|}{ Cause of hypovolemia, $\mathrm{n}(\%)$} \\
\hline Sepsis & $186 / 385(48.3)$ & $183 / 356(51.4)$ \\
\hline Trauma & $60 / 385(15.6)$ & $56 / 356(15.7)$ \\
\hline Other & $139 / 385(36.1)$ & $117 / 356(32.9)$ \\
\hline \multicolumn{3}{|l|}{$\begin{array}{l}\text { Fluid administration before ICU admission (within } \\
\text { the past } 12 \mathrm{~h} \text { ) }\end{array}$} \\
\hline Crystalloids, n (\%) & $269 / 385(70.0)$ & $231 / 356(64.9)$ \\
\hline Dose, median (IQR), ml & $1,000(500-2,500)$ & $1,500(500-2,500)$ \\
\hline Colloids, n (\%) & $266 / 385(69.1)$ & $235 / 356(66.0)$ \\
\hline Dose, median (IQR), ml & $500(0-1,000)$ & $500(0-1,000)$ \\
\hline
\end{tabular}

The Knaus scale is defined as follows: A, prior good health, no functional limitations; B, mild to moderate limitation of activity because of chronic medical problem; $\mathrm{C}$, chronic disease producing serious but not incapacitating restriction of activity; and $\mathrm{D}$, severe restriction of activity caused by disease; includes persons bedridden or institutionalized because of illness.

$\mathrm{ICU}$, intensive care unit; IQR, interquartile range. 
Table 2. Type of Fluid Administered by Randomization Arm (Cumulative Dose Administered over a 7-day Period)

\begin{tabular}{|c|c|c|c|}
\hline & $\begin{array}{l}\text { Colloids Arm } \\
\quad(n=385)\end{array}$ & $\begin{array}{l}\text { Crystalloids Arm } \\
\quad(n=356)\end{array}$ & $P$ \\
\hline Isotonic saline, n (\%) Volume, median (IQR), ml & $\begin{array}{c}70 / 385(18.2) \\
2,000(1,000-4,900)\end{array}$ & $\begin{array}{c}313 / 356(87.9) \\
3,000(1,500-5,500)\end{array}$ & $\begin{array}{l}0.0001 \\
0.050\end{array}$ \\
\hline Ringer's lactate, n (\%) Volume, median (IQR), ml & $\begin{array}{c}36 / 385(9.4) \\
3,500(1,000-6,125)\end{array}$ & $\begin{array}{c}120 / 356(33.7) \\
2,500(1,000-5,000)\end{array}$ & $\begin{array}{l}0.001 \\
0.815\end{array}$ \\
\hline Hypertonic saline, n (\%) Volume, median (IQR), ml & $\begin{array}{c}9 / 385(2.3) \\
750(350-2,500)\end{array}$ & $\begin{array}{c}16 / 356(4.5) \\
1,250(620-2,625)\end{array}$ & $\begin{array}{l}0.462 \\
0.893\end{array}$ \\
\hline Gelatins, n (\%) Volume, median (IQR), ml & $\begin{array}{c}171 / 385(44.4) \\
1,500(1,000-3,500)\end{array}$ & $\begin{array}{c}7 / 356(2.0) \\
500(500-750)\end{array}$ & $\begin{array}{l}0.002 \\
0.098\end{array}$ \\
\hline Hydroxyethyl starch, n (\%) Volume, median (IQR), ml & $\begin{array}{c}299 / 385(77.7) \\
1,500(1,000-2,275)\end{array}$ & $\begin{array}{c}24 / 356(6.7) \\
500(500-1,125)\end{array}$ & $\begin{array}{l}0.004 \\
0.002\end{array}$ \\
\hline Albumin $20 \%, \mathrm{n}(\%)^{\star}$ Volume, median (IQR), ml & $\begin{array}{c}5 / 385(1.3) \\
200(200-300)\end{array}$ & $\begin{array}{c}11 / 356(3.1) \\
330(200-400)\end{array}$ & $\begin{array}{l}0.166 \\
0.832\end{array}$ \\
\hline Albumin 4\%, n (\%)* Volume, median (IQR), ml & $\begin{array}{c}5 / 385(1.3) \\
500(500-500)\end{array}$ & $\begin{array}{c}6 / 356(1.7) \\
725(500-1,362)\end{array}$ & $\begin{array}{l}0.867 \\
0.754\end{array}$ \\
\hline
\end{tabular}

*Administration of albumin to correct hypoalbuminemia (albumin $<20 \mathrm{~g} / \mathrm{l}$ ) was not taken into account.

IQR, interquartile range.

odds ratio, $0.86 ; 95 \% \mathrm{CI}, 0.61$ to $1.21 ; P=0.768$; fig. 3 ). No interaction of the intervention with any of the randomization strata (sepsis, trauma, and other) was found (fig. 4). No interaction of the intervention with the type of surgery was found (fig. 5). There was no difference in the occurrence of death by day 90 between groups: 111 (31.2\%) in the crystalloids arm compared to $122(31.7 \%)$ patients in the colloids arm (adjusted odds ratio, 0.97; 95\% CI, 0.70 to 1.33 ;
$P=0.919$; fig. 6). The time course of the global Sequential Organ Failure Assessment score was similar in both groups $(P=0.915$; fig. 7$)$. The median number of days alive within the first 7 days with a Sequential Organ Failure Assessment score less than 6 did not significantly differ between arms: 2 ( 0 to 4.25 ) days in the crystalloids arm versus 2 ( 0 to 4$)$ days in the colloids arm $(P=0.786)$. Renal replacement therapy was required for 42 patients $(11.8 \%)$ in the crystalloids

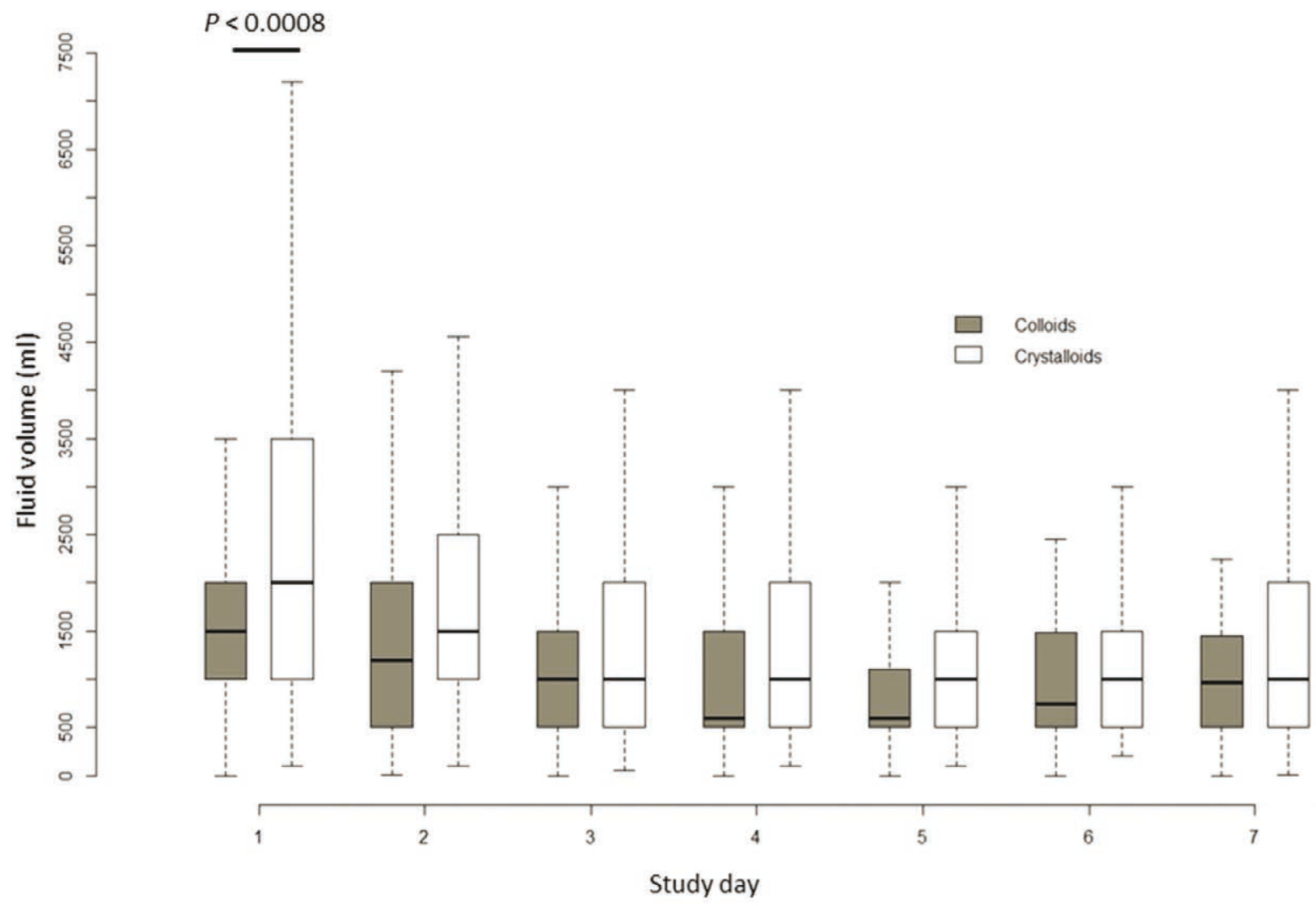

Fig. 2. Total fluid administered over a 7-day period. The amount of colloids administered to achieve hemodynamic stability over the first $24 \mathrm{~h}$ was markedly lower than the amount of crystalloids $(P<0.0008)$. 
arm versus 49 patients (12.7\%) in the colloids arm, over a 7 -day period $(P=0.897)$. The median length of stay in the intensive care unit did not significantly differ: 7 (3 to 17) days in the crystalloids arm compared to 7 (3 to 15) days in the colloids arm $(P=0.855)$. The median length of stay

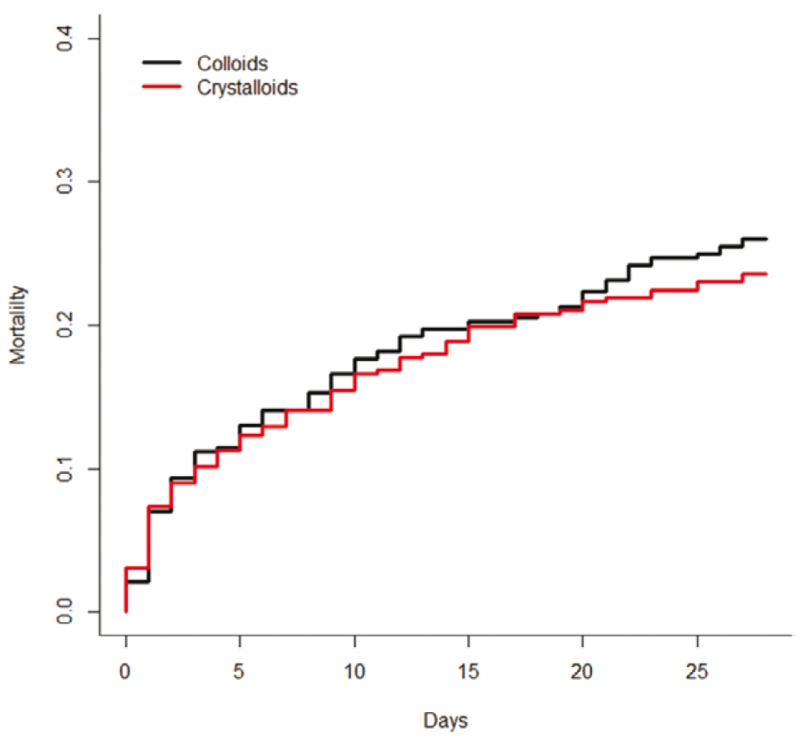

Fig. 3. Mortality over a 28-day period after randomization in the colloid group, compared with the crystalloid group. in the hospital was 22 (11 to 40 ) days in the crystalloids arm compared to 21 (10.5 to 38 ) days in the colloids arm $(P=0.815)$. We also performed a per-protocol comparison of the 340 patients who effectively received colloids and the 259 patients who effectively received crystalloids. Of note, we excluded from the per-protocol analysis all patients in the crystalloid arm who had been treated by albumin even though the protocol provided for albumin supplementation in case of hypoalbuminemia. The per-protocol analysis did not find any difference in the rate of death by day 28 (87 [25.6\%] vs. 54 [20.8\%]; adjusted odds ratio, 0.75; 95\% CI, 0.51 to $1.12 ; P=0.429)$. The results of the per-protocol analysis are in table 3 .

\section{Secondary Surgical Interventions and Blood Transfusion}

Secondary surgery was required in 278 (37.5\%) patients, of which $131(36.8 \%)$ were in the crystalloids group and $147(38.2 \%)$ in the colloids group $(P=0.875)$. A median number of 2.7 (2.2 to 3.4) units of packed erythrocytes were administered to patients in the crystalloids arm compared to 2.7 (2.3 to 3.5$)$ units in the colloids arm $(P=0.890)$. A median number of 2 ( 1 to 5 ) units of platelets was administered to patients in the crystalloids arm compared to 2 (1 to $3)$ units in the colloids arm $(P=0.533)$. A median volume of 450 (175 to 800$) \mathrm{ml}$ of fresh frozen plasma was administered in the crystalloids arm compared to $600(400$ to 1,200$)$

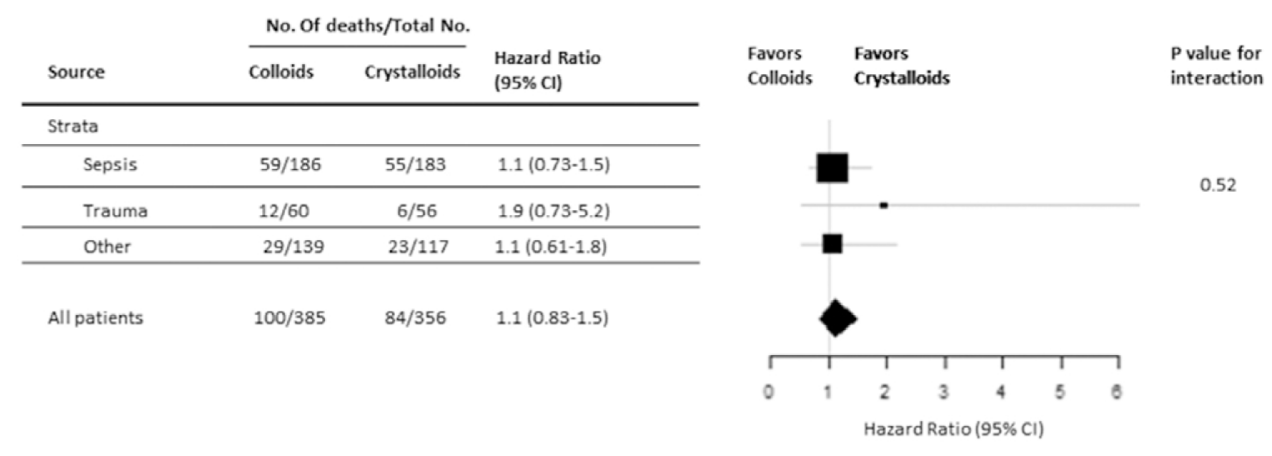

Fig. 4. Hazard ratio for 28-day mortality in the colloid group, compared with the crystalloid group, overall and in predefined subgroups. Size of data markers correspond to the relative size of each subgroup. Error bars indicate $95 \% \mathrm{Cls}$.

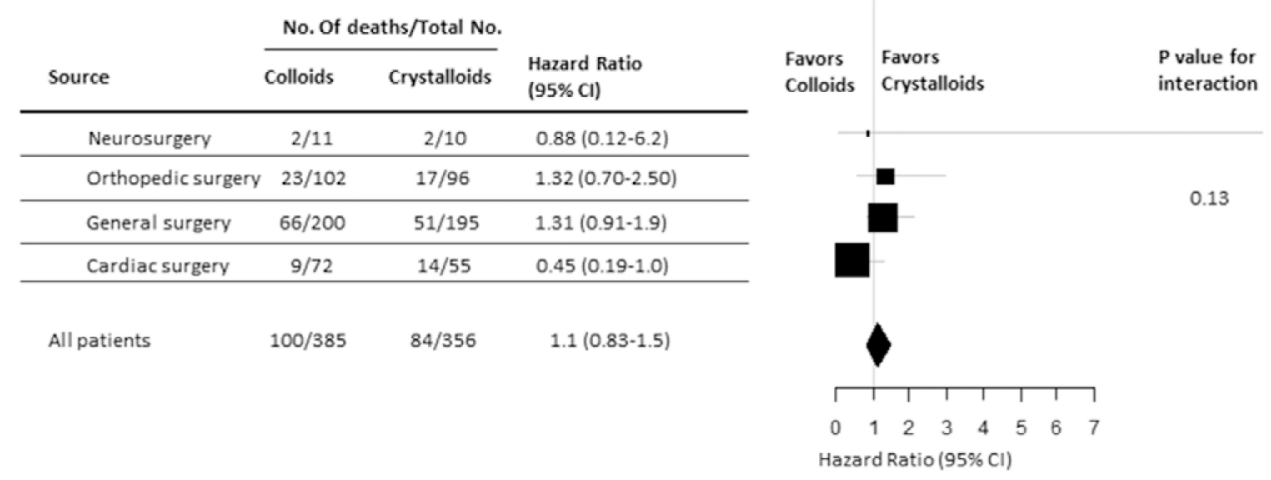

Fig. 5. Hazard ratio for 28-day mortality in the colloid group, compared with the crystalloid group, overall and by type of surgery. Size of data markers correspond to the relative size of each subgroup. Error bars indicate $95 \% \mathrm{Cls}$. 


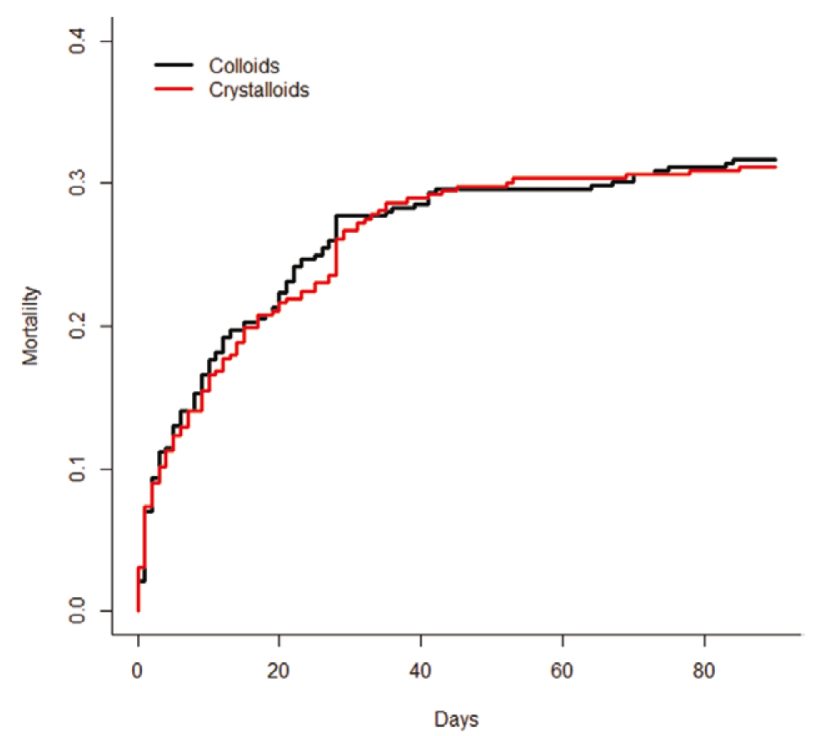

Fig. 6. Mortality over a 90-day period after randomization in the colloid group, compared with the crystalloid group.

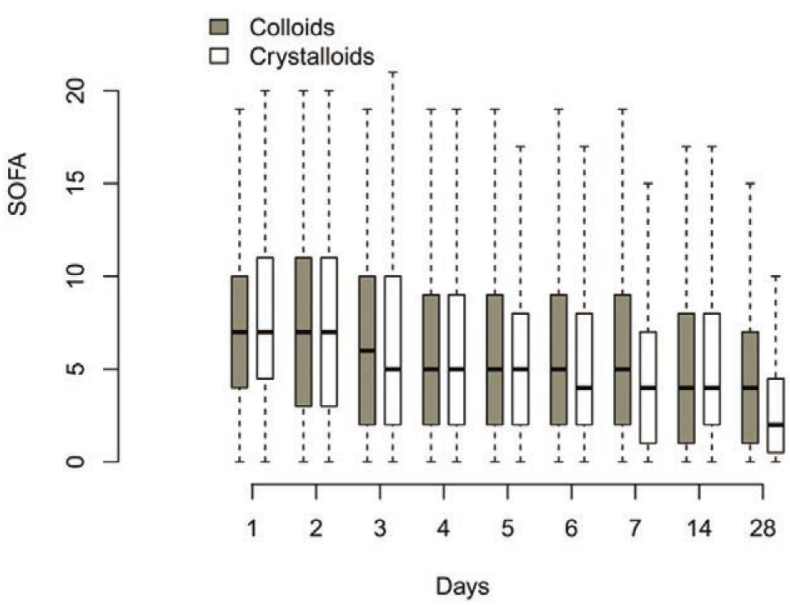

Fig. 7. Global Sequential Organ Failure Assessment score over a 28-day period after randomization in the colloid group, compared with the crystalloid group. Error bars indicate $95 \%$ Cls. SOFA, Sepsis-related Organ Failure Assessment.

$\mathrm{ml}$ in the colloids arm $(P=0.108)$. Additional information regarding the requirement for blood products are found in Supplemental Digital Content, supplemental tables 2 to 7 (http://links.lww.com/ALN/B772). The results of the perprotocol analysis regarding surgical interventions and blood transfusions are found in table 3 .

\section{Discussion}

In this subgroup analysis of a large pragmatic trial comparing the administration of crystalloids to colloids in surgical patients, we found that colloids did not exhibit a significantly different safety profile from crystalloids. The current analysis encompasses both elective and unscheduled surgery associated with clinically significant hypovolemia. Our main finding was that 28-day mortality did not differ between treatment arms, both in the intention-to-treat and the perprotocol analyses. Additionally, mortality by day $28 \mathrm{did}$ not differ in any of the three prespecified strata. Mortality by day 90 did not differ between treatment arms, neither in the intention-to-treat nor in the per-protocol analysis. Inhospital and in-intensive care unit length of stay did not differ between treatment arms. The need for secondary surgical interventions did not significantly differ between groups. The amounts of packed erythrocytes, of platelets, and of fresh frozen plasma did not differ between patients treated by crystalloids and those receiving colloids. Importantly, the global Sequential Organ Failure Assessment score and the need for renal replacement therapy did not differ between groups. Because patients were randomized in CRISTAL to receive products belonging to a broad family of fluids, extrapolating our findings to a particular type of solution, such as balanced solutions, is hazardous. ${ }^{16}$ We did not record enough data to provide information regarding the development of acute kidney injury in this cohort. However, in similar trials, the occurrence of acute kidney injury was found to be inconsistent with other markers of kidney failure, such as the requirements for renal replacement therapy., 5

The whole population of the CRISTAL trial mainly encompassed patients admitted to the intensive care unit for medical reasons $(71 \%){ }^{4}$ In the primary analysis of the CRISTAL trial, death by day 28 did not differ significantly between the colloids and crystalloids groups (relative risk, $0.96 ; 95 \% \mathrm{CI}, 0.88$ to $1.04 ; P=0.26$ ), and mortality by day 90 was significantly lower in the colloids arm (relative risk, 0.92 ; $95 \% \mathrm{CI}, 0.86$ to $0.99 ; P=0.03$ ). In this exploratory analysis of the surgical population, mortality rates by days 28 and 90 are broadly similar to that of the global CRISTAL population, as well as the risk for renal replacement therapy requirement (relative risk, 0.93 ; $95 \% \mathrm{CI}, 0.83$ to 1.03 ; $P=0.19$ ). Data pertaining more specifically to the surgical subpopulation (e.g., bleeding risk and need for secondary surgery) were not analyzed in the whole population of the CRISTAL trial.

\section{Colloids for the Surgical and the Trauma Patient}

The most thoroughly studied subtype of colloid in the surgical context is starch. Retrospective studies hint at the possibility of acute kidney injury after the administration of starches in the surgical setting. ${ }^{17}$ However, several small randomized controlled trials in elective surgery reported no renal side effect related to hydroxyethyl starch administration. ${ }^{18,19}$ In the Crystalloid versus Hydorxyethyl Starch Trial, the number of surgical patients undergoing renal replacement therapy did not differ between both groups: 61 of 1,425 assigned to hydroxyethyl starch (4.3\%) versus 45 of 1,447 assigned to saline (3.0\%) (relative risk, 1.38 ; $95 \%$ CI, 0.94 to 2.01 ).${ }^{20}$ The Fluids in Resuscitation of Severe Trauma trial, comparing the administration of starches to that of crystalloids in both blunt and penetrating trauma, found no difference in mortality, 


\begin{tabular}{lrr} 
& $\begin{array}{c}\text { Colloids Arm } \\
(\mathrm{n}=340)\end{array}$ & $\begin{array}{c}\text { Crystalloids Arm } \\
(\mathrm{n}=259)\end{array}$ \\
\hline Death by day 28, $\mathrm{n}(\%)$ & $87 / 340(25.6)$ & $54 / 259(20.9)$ \\
Death by day 90, $\mathrm{n}(\%)$ & $109 / 340(32.1)$ & $74 / 259(28.6)$ \\
Renal replacement therapy, $\mathrm{n}(\%)$ & $48 / 340(14.1)$ & $27 / 259(10.4)$ \\
Length of stay in the ICU, median (IQR), days & $7(3-14)$ & $6(3-15)$ \\
Length of stay in the hospital, median (IQR), days & $21(10-38)$ & $20(11-38)$ \\
Secondary surgery requirement, n (\%) & $132 / 340(38.8)$ & 0.745 \\
Packed erythrocyte transfusion, median (IQR), units & $2(2-4)$ & 0.891 \\
Platelet transfusion, median (IQR), units & $2(1-3)$ & 0.905 \\
Fresh frozen plasma administration, median (IQR), ml & $600(400-1,088)$ & 0.870 \\
\hline
\end{tabular}

$I C U$, intensive care unit; IQR, interquartile range.

whereas acute kidney injury occurred more frequently in the saline group. ${ }^{21} \mathrm{~A}$ recent trial found that the use of colloids was associated with fewer complications than use of crystalloids in elective abdominal surgery. ${ }^{22}$ Several meta-analyses of surgical patients concluded that starches do not induce additional renal injury. ${ }^{23-25}$ Although these analyses have been criticized, ${ }^{26,27}$ our findings, namely no increased need for renal replacement therapy associated with the administration of colloids, are in keeping with previous reports. Such a difference in the occurrence of acute kidney injury between subjects affected by sepsis and those affected by surgery or trauma may be related to different pathophysiology of acute kidney injury. If blood loss from trauma or during surgery leads to hypotension and renal ischemia, prompt restoration of renal hemodynamic may reduce the incidence of acute kidney injury. ${ }^{28}$ The pathophysiology of sepsis-associated acute kidney injury is wholly different, because acute kidney injury may occur despite normal renal blood flow. ${ }^{29}$ Pathophysiologic mechanisms of sepsisassociated acute kidney injury include inflammation, microcirculatory dysfunction, and endothelial cell injury. ${ }^{30}$ Starches may also affect bleeding in surgical patients. Several trials comparing starches to crystalloids found that starches reduced the clot strength and increased bleeding during both major surgery and cardiac surgery. ${ }^{31-33}$ In the Fluids in Resuscitation of Severe Trauma trial, patients suffering from blunt trauma randomized to the hydroxyethyl starch group required significantly more blood products than those randomized to receive saline. ${ }^{21}$ We did not observe any difference in the required amount of packed erythrocytes, platelets, or fresh frozen plasma. Little is known of the effect of colloid versus crystalloid solutions on mortality in the surgical setting, because surgical-related mortality in most circumstances is extremely low. In the subgroup of patients undergoing surgery before randomization into the $6 \mathrm{~S}$ trial, death by day 90 occurred in 61 of 131 patients in the hydroxyethyl starch subgroup versus 53 of 146 patients in the Ringer's acetate subgroup (relative risk, $1.28 ; 95 \%$ CI, 0.97 to $1.70 ; P=0.42) .{ }^{34}$ A last point to be mentioned when comparing crystalloids to colloids is the cost of each product. CRISTAL was not designed to analyze the cost of both interventions.
No power computation was performed when this secondary analysis of the trial was undertaken. Nevertheless, given the sample size of 741 patients broadly divided into two equal-sized groups, the statistical power to detect an effect size of at least 0.2 was above $80 \%$. Strengths of the current study include the fact that our data set stems from a pragmatic, randomized clinical trial, depicting the use of resuscitation fluids in real-world conditions. Additionally, our analyses were preplanned, and we report on a small number of outcomes, reducing the risk of false-positive results. ${ }^{35}$ Our study has several limitations. First, we did not have access to perioperative blood loss; we therefore had to use a surrogate marker of blood loss, the number of packed erythrocytes administered. Second, because our population consists of patients who were transferred from the operating theater to the intensive care unit, extrapolation of our findings to all patients managed in the operating theater requires careful consideration.

\section{Conclusions}

In surgical patients included in the CRISTAL trial, we found no difference between colloids and crystalloids regarding safety, namely the risk of death or of organ failure, including acute kidney injury. The safety of colloids was comparable to that of crystalloids in our population of surgical patients treated for hypovolemic shock.

\section{Acknowledgments}

The authors thank Julie Lejeune, M.S. (Biostatistical Unit, Saint Louis Hospital, Paris, France), for her technical assistance, as well as all the investigators and patients of the CRISTAL trial.

\section{Research Support}

Supported in 2001 and 2010 by grant No. AOM 01020 from the French Ministry of Health.

\section{Competing Interests}

The authors declare no competing interests. 


\section{Correspondence}

Address correspondence to Dr. Annane: General Intensive Care Unit, Raymond Poincaré Hospital (AP-HP), University of Versailles Saint-Quentin en Yvelines 104, Boulevard Raymond Poincaré 92380, Garches, France. djillali.annane@ aphp.fr. Information on purchasing reprints may be found at www.anesthesiology.org or on the masthead page at the beginning of this issue. Anesthesiology's articles are made freely accessible to all readers, for personal use only, 6 months from the cover date of the issue.

\section{References}

1. Bellomo R, Bion J, Finfer S, Myburgh J, Perner A, Reinhart $\mathrm{K}$; all co-signatories: Open letter to the Executive Director of the European Medicines Agency concerning the licensing of hydroxyethyl starch solutions for fluid resuscitation. Br J Anaesth 2014; 112:595-600

2. Coriat P, Guidet B, de Hert S, Kochs E, Kozek S, Van Aken $\mathrm{H}$; all co-signatories listed online: Counter statement to open letter to the Executive Director of the European Medicines Agency concerning the licensing of hydroxyethyl starch solutions for fluid resuscitation. Br J Anaesth 2014; 113:194-5

3. Brunkhorst FM, Engel C, Bloos F, Meier-Hellmann A, Ragaller $\mathrm{M}$, Weiler N, Moerer O, Gruendling M, Oppert M, Grond S, Olthoff D, Jaschinski U, John S, Rossaint R, Welte T, Schaefer M, Kern P, Kuhnt E, Kiehntopf M, Hartog C, Natanson C, Loeffler M, Reinhart K; German Competence Network Sepsis (SepNet): Intensive insulin therapy and pentastarch resuscitation in severe sepsis. N Engl J Med 2008; 358:125-39

4. Annane D, Siami S, Jaber S, Martin C, Elatrous S, Declère AD, Preiser JC, Outin H, Troché G, Charpentier C, Trouillet JL, Kimmoun A, Forceville X, Darmon M, Lesur O, Reignier J, Régnier J, Abroug F, Berger P, Clec'h C, Cle'h C, Cousson J, Thibault L, Chevret S; CRISTAL Investigators: Effects of fluid resuscitation with colloids $v s$. crystalloids on mortality in critically ill patients presenting with hypovolemic shock: The CRISTAL randomized trial. JAMA 2013; 310:1809-17

5. Myburgh JA, Finfer S, Bellomo R, Billot L, Cass A, Gattas D, Glass P, Lipman J, Liu B, McArthur C, McGuinness S, Rajbhandari D, Taylor CB, Webb SA; CHEST Investigators; Australian and New Zealand Intensive Care Society Clinical Trials Group: HydroxyCethyl starch or saline for fluid resuscitation in intensive care. N Engl J Med 2012; 367:1901-11

6. European Medicines Agency: PRAC confirms that hydroxyethyl-starch solutions (HES) should no longer be used in patients with sepsis or burn injuries or in critically ill patients. Available at: http://www.ema.europa.eu/docs/en_GB/document_library/Press_release/2013/10/WC500151964.pdf. Accessed May 7, 2018

7. Perner A, Haase N, Guttormsen AB, Tenhunen J, Klemenzson G, Åneman A, Madsen KR, Møller MH, Elkjær JM, Poulsen LM, Bendtsen A, Winding R, Steensen M, Berezowicz P, SøeJensen P, Bestle M, Strand K, Wiis J, White JO, Thornberg KJ, Quist L, Nielsen J, Andersen LH, Holst LB, Thormar K, Kjældgaard AL, Fabritius ML, Mondrup F, Pott FC, Møller TP, Winkel P, Wetterslev J; 6S Trial Group; Scandinavian Critical Care Trials Group: Hydroxyethyl starch 130/0.42 versus Ringer's acetate in severe sepsis. N Engl J Med 2012; 367:124-34

8. Scheingraber S, Rehm M, Sehmisch C, Finsterer U: Rapid saline infusion produces hyperchloremic acidosis in patients undergoing gynecologic surgery. ANESTHESIOLOGY 1999; 90:1265-70

9. Chowdhury AH, Cox EF, Francis ST, Lobo DN: A randomized, controlled, double-blind crossover study on the effects of 2-L infusions of $0.9 \%$ saline and plasma-lyte ${ }^{\circledR} 148$ on renal blood flow velocity and renal cortical tissue perfusion in healthy volunteers. Ann Surg 2012; 256:18-24
10. McCabe WR, Jackson GG: Gram-negative bacteremia: I. Etiology and ecology. Arch Intern Med 1962; 110: 847-55

11. Knaus WA, Zimmerman JE, Wagner DP, Draper EA, Lawrence DE: APACHE-acute physiology and chronic health evaluation: A physiologically based classification system. Crit Care Med 1981; 9:591-7

12. Le Gall JR, Lemeshow S, Saulnier F: A new Simplified Acute Physiology Score (SAPS II) based on a European/North American multicenter study. JAMA 1993; 270:2957-63

13. Vincent JL, Moreno R, Takala J, Willatts S, De Mendonça A, Bruining H, Reinhart CK, Suter PM, Thijs LG: The SOFA (Sepsis-related Organ Failure Assessment) score to describe organ dysfunction/failure: On behalf of the Working Group on Sepsis-Related Problems of the European Society of Intensive Care Medicine. Intensive Care Med 1996; 22:707-10

14. Baker SP, O'Neill B, Haddon W Jr, Long WB: The Injury Severity Score: A method for describing patients with multiple injuries and evaluating emergency care. J Trauma 1974; 14:187-96

15. Benjamini Y, Hochberg Y: Controlling the false discovery rate: A practical and powerful approach to multiple testing. $J$ R Stat Soc Ser B Methodol 1995; 57:289-300

16. Yunos NM, Bellomo R, Hegarty C, Story D, Ho L, Bailey M: Association between a chloride-liberal vs chloride-restrictive intravenous fluid administration strategy and kidney injury in critically ill adults. JAMA 2012; 308:1566-72

17. Kashy BK, Podolyak A, Makarova N, Dalton JE, Sessler DI, Kurz A: Effect of hydroxyethyl starch on postoperative kidney function in patients having noncardiac surgery. ANESTHESIOLOGY 2014; 121:730-9

18. Kancir AS, Pleckaitiene L, Hansen TB, Ekeløf NP, Pedersen EB: Lack of nephrotoxicity by $6 \%$ hydroxyethyl starch 130/0.4 during hip arthroplasty: A randomized controlled trial. ANESTHESIOLOGY 2014; 121:948-58

19. Kancir AS, Johansen JK, Ekeloef NP, Pedersen EB: The effect of $6 \%$ hydroxyethyl starch $130 / 0.4$ on renal function, arterial blood pressure, and vasoactive hormones during radical prostatectomy: A randomized controlled trial. Anesth Analg 2015; 120:608-18

20. Wilkes M, Navickis, RJ: Postoperative renal replacement therapy after hydroxyethyl starch infusion: A meta-analysis of randomised trials. Neth J Crit Care 2014:4-9

21. James MF, Michell WL, Joubert IA, Nicol AJ, Navsaria PH, Gillespie RS: Resuscitation with hydroxyethyl starch improves renal function and lactate clearance in penetrating trauma in a randomized controlled study: The FIRST trial (Fluids in Resuscitation of Severe Trauma). Br J Anaesth 2011; 107:693-702

22. Joosten A, Delaporte A, Ickx B, Touihri K, Stany I, Barvais L, Van Obbergh L, Loi P, Rinehart J, Cannesson M, Van der Linden P: Crystalloid versus colloid for intraoperative goaldirected fluid therapy using a closed-loop system: A randomized, double-blinded, controlled trial in major abdominal surgery. Anesthesiology 2018; 128:55-66

23. Martin C, Jacob M, Vicaut E, Guidet B, Van Aken H, Kurz A: Effect of waxy maize-derived hydroxyethyl starch 130/0.4 on renal function in surgical patients. ANESTHESIOLOGY 2013; 118:387-94

24. Van Der Linden P, James M, Mythen M, Weiskopf RB: Safety of modern starches used during surgery. Anesth Analg 2013; 116:35-48

25. Jacob M, Fellahi JL, Chappell D, Kurz A: The impact of hydroxyethyl starches in cardiac surgery: A meta-analysis. Crit Care 2014; 18:656

26. Groeneveld AB, Navickis RJ, Wilkes MM: Hydroxyethyl starch 130/0.4 and postoperative acute kidney injury. ANESTHESIOLOGY 2013; 119:733-5 
27. Takala J, Hartog C, Reinhart K: Safety of modern starches used during surgery: Misleading conclusions. Anesth Analg 2013; 117:527-8

28. Goren O, Matot I: Perioperative acute kidney injury. Br J Anaesth 2015; 115:ii3-14

29. Zarbock A, Gomez H, Kellum JA: Sepsis-induced acute kidney injury revisited: Pathophysiology, prevention and future therapies. Curr Opin Crit Care 2014; 20:588-95

30. Ergin B, Kapucu A, Demirci-Tansel C, Ince C: The renal microcirculation in sepsis. Nephrol Dial Transplant 2015; 30:169-77

31. Rasmussen KC, Johansson PI, Højskov M, Kridina I, Kistorp T, Thind P, Nielsen HB, Ruhnau B, Pedersen T, Secher NH: Hydroxyethyl starch reduces coagulation competence and increases blood loss during major surgery: Results from a randomized controlled trial. Ann Surg 2014; 259:249-54
32. Skhirtladze K, Base EM, Lassnigg A, Kaider A, Linke S, Dworschak M, Hiesmayr MJ: Comparison of the effects of albumin 5\%, hydroxyethyl starch 130/0.4 6\%, and Ringer's lactate on blood loss and coagulation after cardiac surgery. Br J Anaesth 2014; 112:255-64

33. Schramko A, Suojaranta-Ylinen R, Kuitunen A, Raivio P, Kukkonen S, Niemi T: Hydroxyethylstarch and gelatin solutions impair blood coagulation after cardiac surgery: A prospective randomized trial. Br J Anaesth 2010; 104:691-7

34. Müller RG, Haase N, Wetterslev J, Perner A: Effects of hydroxyethyl starch in subgroups of patients with severe sepsis: Exploratory post-hoc analyses of a randomised trial. Intensive Care Med 2013; 39:1963-71

35. Sun X, Ioannidis JP, Agoritsas T, Alba AC, Guyatt G: How to use a subgroup analysis: Users' guide to the medical literature. JAMA 2014; 311:405-11 